\title{
Fistula Formation 6 Years after Removal of Infected Pacemaker Leads
}

\author{
Thomas Schroeter $^{1}$ Philipp Kiefer ${ }^{1}$ Matthias Sauer ${ }^{1}$ Friedrich Wilhelm Mohr ${ }^{1}$ \\ ${ }^{1}$ Department of Cardiac Surgery, Heart Center Leipzig, University of
Leipzig, Leipzig, Germany \\ Address for correspondence Thomas Schroeter, MD, Department of \\ Cardiac Surgery, Heart Center Leipzig, Struempellstrasse 39, Leipzig \\ 04289, Germany (e-mail: thomas-schroeter@gmx.de).
} Thorac Cardiovasc Surg Rep 2015;4:49-51.

\begin{abstract}
Keywords

- endocarditis

- pacemaker

- fistula

- infection

We describe a case of a male patient who presented with a chronic ulcer below the left clavicle. Six years before the present admission a permanent pacemaker, including leads, was explanted related to endocarditis. The initial working hypothesis suspected an infected sebaceous gland as the cause of ulceration. After two periods of unsuccessful surgical treatment of the gland, further examination identified a small pacemaker lead fragment underneath the articulation between sternum and clavicle as a possible reason.
\end{abstract}

\section{Introduction}

In case of infective endocarditis, removal of permanent pacemakers (PPMs) and leads is a recommended procedure by the professional associations. ${ }^{1-3}$ However, explantation of the leads can sometimes be difficult due to adhesions and may remain incomplete. A percutaneous catheter-based removal of remnants resting in the vascular system is difficult but feasible. Various snares, forceps, or baskets can be used. However, if the fragment is located outside the vascular system in elusive regions, fragments are sometimes left in situ. Usually this is possible without further problems, but in very rare cases it can lead to the formation of fistulas and chronic wounds as a delayed reaction.

\section{Case Presentation}

We report a case of a 68-year-old male patient, who was admitted to our hospital with recurrent redness, pain, and purulent secretion of a small ulcer under the left collarbone. His medical history revealed a complete atrioventricular block, which was diagnosed in 2003 and treated by implantation of a PPM. In 2006, the system had to be removed due to lead-associated endocarditis; thereby, a small fragment remained in the right ventricle. In addition, a lead remnant of the old ventricular lead remained below the left clavicle. A part of the right ventricular fragment could be removed using a Snare Kit (Needle's Eye Snar; William Cook Europe, Bjaeverskov, Denmark); the remnant under the clavicle was left in situ. In addition to these findings, the patient suffers from arterial hypertension, a slightly reduced left ventricular function with an ejection fraction of $45 \%$ and a low-grade coronary atherosclerosis without significant lesions.

At the first admission to the hospital in April 2012, the patient presented with a small subclavian ulceration. Under suspicion of an abscess of a sebaceous gland, a surgical division and local disinfectant treatment was done. A few weeks later, this procedure had to be repeated due to reulceration.

In early 2013 , the patient was readmitted to the hospital with same complaints. There were local swelling, tenderness, and purulent secretion. Signs of systemic infection could not be detected. We suspected an 8-cm-long fistula between the lead remnant under the left collarbone, seen 6 years ago, and the skin surface (-Fig. 1). Based on this assumption, we decided to resect the fistula and remove the fragment.

The procedure was performed under general anesthesia. In the first step, the lead tip was localized by fluoroscopy and the area in which the fragment was projected was marked on the skin surface (-Fig. 2). We used Siemens Artis Zeego multiaxis system (Siemens Healthcare, Erlangen, Germany) for screening to permit localization in several angulations. The next step was to explore the fistula by a small probe, followed by spindle-shaped cutting of the same. Because of severe received

July 24, 2014

accepted after revision

September 10, 2014

published online

February 17, 2015
DOI http://dx.doi.org/

10.1055/s-0034-1395166. ISSN 2194-7635.
@ 2015 Georg Thieme Verlag KG
Stuttgart · New York

License terms

(1) $\circledast \circledast$ 


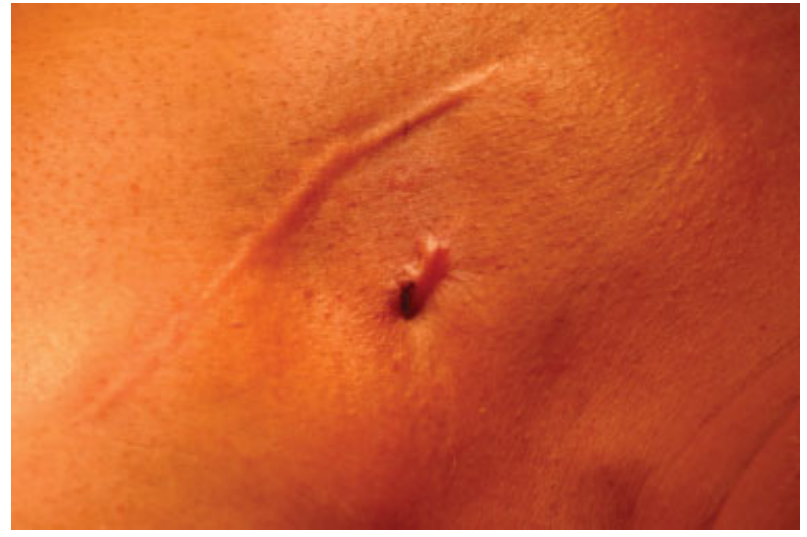

Fig. 1 In addition to the scar of the old permanent pacemaker pocket the ulceration of the fistula is obvious.

adhesions, a further supraclavicular incision was necessary to reach the fragment (-Fig. 3). Another advantage of the additional supraclavicular incision was facilitated access to the lead remnant and better control in case of hemorrhagic complications. In addition, the strong connective tissue of the fistula served as a guidance structure to reach and mobilize the fistula and the lead fragment. We were able to rescue a $10 \mathrm{~cm}$ lead isolation fragment, the anchor, and the electrode tip of the pacemaker lead explanted 6 years ago ( - Fig. 4). This finding was unexpected, because the silicone insulation of the pacing lead was invisible under fluoroscopy and we had assumed only a lead tip or a small fragment. The fistula ended right on the subclavian vein. To prevent oozing, we placed a Tabotamp hemostat (TABOTAMP, Ethicon Biosurgery, Johnson \& Johnson Medical GmbH, Norderstedt, Germany), a densely woven knit of oxidized regenerated cellulose, inside

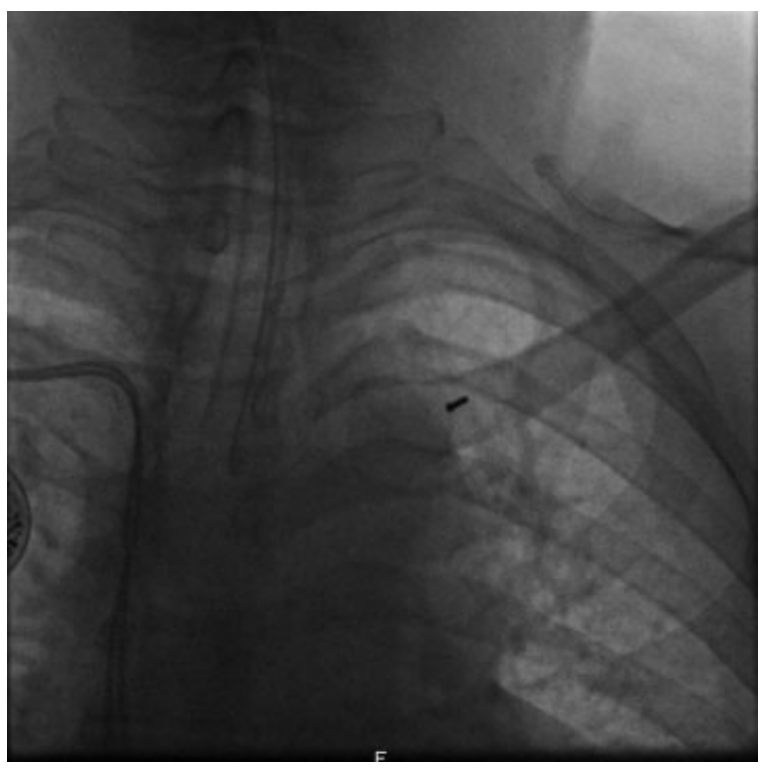

Fig. 2 Chest X-ray: Under the left clavicle, the remaining electrode tip is visible. In addition, the endotracheal tube and the right-side permanent pacemakers are shown.

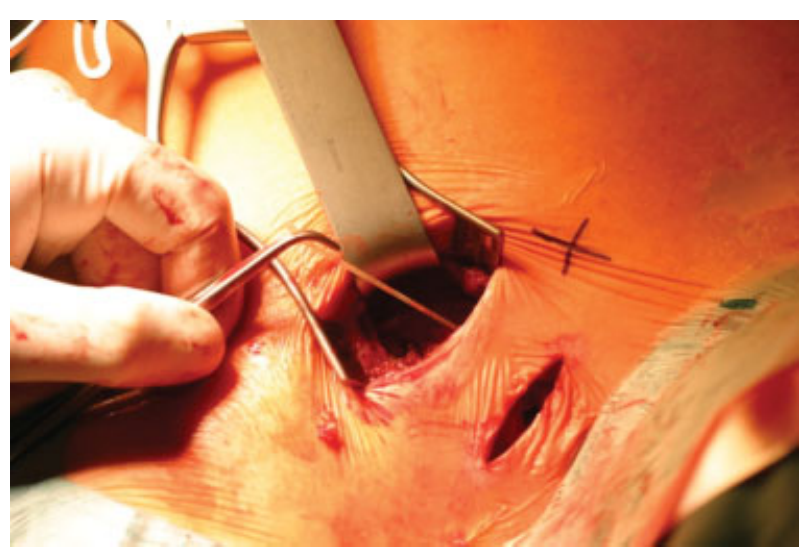

Fig. 3 The image shows the infraclavicular and the supraclavicular access and the remaining lead. The fistula was removed.

the former fistula. To prevent local defense infection, a Sulmycin sponge was furthermore placed (Sulmycin Implant E $5 \times 5 \mathrm{~cm}$, Eusa Pharma GmbH, Munich, Germany).

Postoperative course was uneventful and we released the patient 3 days after the surgery without local or systemic signs of infection. The chest X-ray revealed no further lead remnant in the area of the clavicle.

\section{Conclusion}

The removal of implanted PPM and pacemaker leads in case of endocarditis is recommended by professional societies and expert panels. The described mortality for the complete removal is low in centers with extensive experience and reinfection is rarely observed ${ }^{4-6} \mathrm{~A}$ prerequisite for a successful therapy, however, is the complete removal of all prosthetic material. In addition, infective remnants may complicate the implantation of a PPM on the contralateral side and may cause reinfection of the new system. ${ }^{7,8}$ Nevertheless, a reinfection 6 years following initial surgery is in our experience unique. In the described case it was concluded that a complete removal of inactive or infected leads is necessary. A high success rate requires that the surgeon or cardiologist has a lot

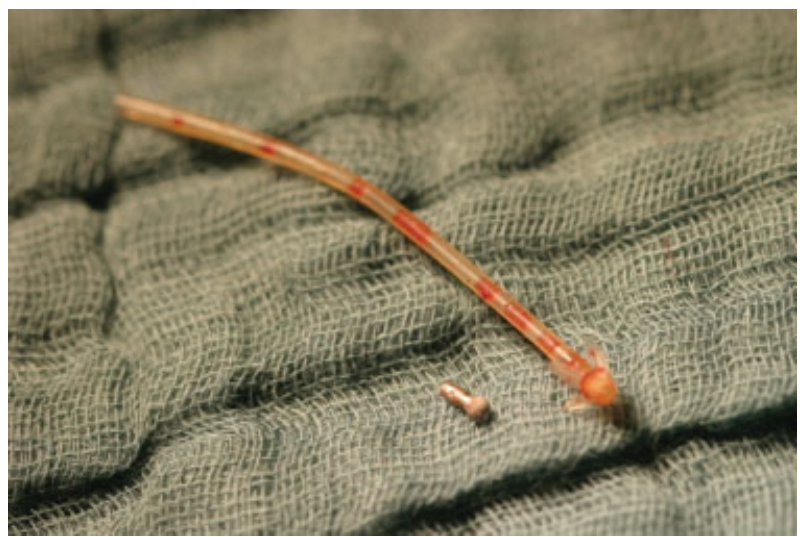

Fig. 4 The removed lead remnant and electrode tip. 
of experience and expertise in this topic. In case of abandoned or broken lead fragments an accurate documentation and optionally a second surgical intervention is necessary to prevent late complications.

\section{References}

1 Wilkoff BL, Love CJ, Byrd CL, et al; Heart Rhythm Society American Heart Association. Transvenous lead extraction: Heart Rhythm Society expert consensus on facilities, training, indications, and patient management: this document was endorsed by the American Heart Association (AHA). Heart Rhythm 2009;6(7):1085-1104

2 Post JJ, Alexopoulos C, Fewtrell C, Giles R, Jones PD. Outcome after complete percutaneous removal of infected pacemaker systems and implantable cardiac defibrillators. Intern Med J 2006;36(12):790-792

3 Sohail MR. Management of infected pacemakers and implantable cardioverter-defibrillators. Intern Med J 2007;37(7):509-510, author reply 510
4 Sohail MR, Uslan DZ, Khan AH, et al. Infective endocarditis complicating permanent pacemaker and implantable cardioverter-defibrillator infection. Mayo Clin Proc 2008;83(1): 46-53

5 Pichlmaier M, Knigina L, Kutschka I, et al. Complete removal as a routine treatment for any cardiovascular implantable electronic device-associated infection. J Thorac Cardiovasc Surg 2011;142(6): $1482-1490$

6 Rastan AJ, Doll N, Walther T, Mohr FW. Pacemaker dependent patients with device infection-a modified approach. Eur J Cardiothorac Surg 2005;27(6):1116-1118

7 Sohail MR, Hussain S, Le KY, et al; Mayo Cardiovascular Infections Study Group. Risk factors associated with early- versus late-onset implantable cardioverter-defibrillator infections. J Interv Card Electrophysiol 2011;31(2):171-183Erratum in: J Interv Card Electrophysiol. 2012;35(3):355. Virkram, H R [corrected to Vikram, HR]

8 Catanchin A, Murdock CJ, Athan E. Pacemaker infections: a 10-year experience. Heart Lung Circ 2007;16(6):434-439 